\title{
Contractive Interference Functions and Rates of Convergence of Distributed Power Control Laws
}

\author{
Hamid Reza Feyzmahdavian, Mikael Johansson and Themistoklis Charalambous
}

\begin{abstract}
The standard interference functions introduced by Yates have been very influential on the analysis and design of distributed power control laws. While powerful and versatile, the framework has some drawbacks: the existence of fixed-points has to be established separately, and no guarantees are given on the rate of convergence of the iterates. This paper introduces contractive interference functions, a slight reformulation of the standard interference functions that guarantees existence and uniqueness of fixed-points and geometric convergence rates. We show that many power control laws from the literature are contractive and derive, sometimes for the first time, convergence rate estimates for these algorithms. Finally, we show that although standard interference functions are not contractive, they are paracontractions with respect to a certain metric space. Extensions to two-sided scalable interference functions are also discussed.
\end{abstract}

\section{INTRODUCTION}

Distributed power control (DPC) algorithms such as [1]-[3] have had an enormous influence on modern wireless systems. The basic algorithm for adjusting transmit powers to meet predefined Signal-to-Interference-and-Noise-Ratio (SINR) targets can be written as a linear iteration and has been thoroughly analyzed. In particular, when the SINR targets are feasible, the algorithm converges to a unique fixed-point at a geometric rate. These results can be derived using Perron-Frobenius theory for positive matrices or, alternatively, by showing that the linear iteration is a contraction mapping in a weighted maximum norm; (e.g. [3], [4]).

An elegant axiomatic framework for studying more general power control iterations was proposed by Yates [5]. The so-called standard interference functions include the linear iterations, and several important nonlinear power control laws. While several results exist for synchronous and asynchronous convergence of standard interference function iterations, the current proofs are tailor-made and the link to contraction mappings, that is essential for the analysis of the linear iterations, is absent. In addition very few results on the convergence rate of such algorithms have appeared in the literature (e.g. [6]-[8] for exceptions). This paper tries to fill this gap.

Contrary to various claims in the literature, we demonstrate that standard interference functions are, in general, not contraction mappings. However, we show that a slight modification of the scalability axiom of standard interference function allows to guarantee contractivity of the iterations and hence unique fixed-points and geometric convergence rates. This

H. R. Feyzmahdavian, M. Johansson and T. Charalambous are with ACCESS Linnaeus Center, School of Electrical Engineering, KTH-Royal Institute of Technology, SE-100 44 Stockholm, Sweden. E-mails: \{hamidrez, mikaelj, themisc\}@kth.se. condition is satisfied by the basic DPC algorithm and allows to recover the same convergence rate that comes out of a tailored analysis. It also allows to estimate the convergence rate of the other power control schemes considered by Yates framework, as well as the utility-based power control scheme developed in [9]. We also demonstrate how a logarithmic change-ofvariables render interference functions Para-contractions. Various extensions of the basic framework have been proposed by Sung and Leung [10] and Schubert and Boche [11]. We give analogous conditions that guarantee that two-sided scalable interference functions [10] define contraction mappings, and hence have unique fixed points and geometric convergence rates. This result is related to the work by Möller and Jönsson [12], who demonstrated that in logarithmic variables, two-sided scalability implies global Lipschitz continuity of the interference function, and an alternative restriction allows to establish convergence rates and uniqueness of fixed-points. Finally, we discuss how asynchronous convergence can be established in our framework. Due to space constraints, we refer the reader to the full-length version of our paper [13] for technical details, omitted proofs and extensions.

Throughout the paper, we use the following notation: vectors are written in bold lower case letters. The $i^{\text {th }}$ component of a vector $\mathbf{x}$ is denoted by $x_{i}$. The notation $\mathbf{x} \geq \mathbf{y}$ implies that $x_{i} \geq y_{i}$ for all elements $i$. We use the notation $\mathbf{x} \geq \mathbf{0}$ to show that all of the components of $\mathbf{x}$ are greater than zero. Let $\mathbf{e}^{\mathbf{x}}$ and $\ln (\mathbf{x})$ denote component-wise exponential and logarithm of the entries $\mathbf{x}$, respectively. Given a vector $\mathbf{v}>\mathbf{0},\|\cdot\|_{\infty}^{v}$ stands for the weighted maximum norm, i.e. $\|\mathbf{x}\|_{\infty}^{v}=\max _{i}\left|x_{i} / v_{i}\right|$. The vector norm $\|\cdot\|_{\infty}^{v}$ induces a matrix norm, also denoted by $\|\cdot\|_{\infty}^{v}$ defined by

$$
\|M\|_{\infty}^{v}=\max _{\mathbf{x} \neq 0} \frac{\|M \mathbf{x}\|_{\infty}^{v}}{\|\mathbf{x}\|_{\infty}^{v}}
$$

When $v_{i}=1$ for each $i$, we suppress the superscript $v$. We use $\rho(A)$ to denote the spectral radius of matrix $A$.

\section{FIXED-POINT THEORY AND INTERFERENCE FUNCTIONS}

\section{A. Fixed-points, contractions and para-contractions}

We consider iterative algoritms on the form

$$
\mathbf{x}(n+1)=T(\mathbf{x}(n)), \quad n=0,1,2, \ldots
$$

where $T$ is a mapping from a subset $X$ of $\mathbb{R}^{n}$ into itself. A vector $\mathbf{x}^{\star}$ is called a fixed point of $T$ if $T\left(\mathbf{x}^{\star}\right)=\mathbf{x}^{\star}$. If $T$ is continuous at $\mathbf{x}^{\star}$ and the sequence $\{\mathbf{x}(n)\}$ converges to $\mathbf{x}^{\star}$, then $\mathbf{x}^{\star}$ is a fixed point of $T$. Therefore, the iteration (1) can 
be viewed as an algorithm for finding such a fixed point of $T$. If $T$ has the following property

$$
\|T(\mathbf{x})-T(\mathbf{y})\| \leq c\|\mathbf{x}-\mathbf{y}\|
$$

where $\|\cdot\|$ is some norm and $c \in[0,1)$, then $T$ is called a contraction mapping. The following proposition shows that contraction mappings have a unique fixed point and geometric convergence rate.

Proposition 1 (Convergence of Contracting Iterations [14]) If $T$ is a contraction mapping in $X$, then:

- (Existence and Uniqueness of Fixed Points) The mapping $T$ has a unique fixed point $\mathrm{x}^{\star} \in X$.

- (Geometric Convergence) For every initial vector $\mathbf{x}(0) \in$ $X$, the sequence $\{\mathbf{x}(n)\}$ generated by $\mathbf{x}(n+1)=$ $T(\mathbf{x}(n))$ converges to $\mathbf{x}^{\star}$ geometrically. In particular,

$$
\left\|\mathbf{x}(n)-\mathbf{x}^{\star}\right\| \leq c^{n}\left\|\mathbf{x}(0)-\mathbf{x}^{\star}\right\|
$$

An operator $T$ on $X$ is called para-contraction if

$$
\|T(\mathbf{x})-T(\mathbf{y})\|<\|\mathbf{x}-\mathbf{y}\| \text { for all } \mathbf{x} \neq \mathbf{y}
$$

Para-contractions have at most one fixed point [14] and, in contrast to contractions, may not have a fixed point. As an example, consider the para-contracting function $T(x)=x+$ $e^{-x}$ in $[0, \infty)$. It is easily seen that $T$ has no fixed point. We also note that para-contractivity does not yield any estimate on the rate of convergence to the fixed point.

\section{B. Standard interference functions}

The interference function framework was introduced by Yates [5] to study various extensions of the basic distributed power control problem of adjusting transmit powers to meet prespecified signal-to-interference and noise ratios.

Definition 1 (Standard Interference Function [5]) A function $\mathbf{I}(\mathbf{p})$ is called standard interference function, if for all $\mathbf{p} \geq \mathbf{0}$ the following properties are satisfied:

- Positivity: $\mathbf{I}(\mathbf{p})>\mathbf{0}$.

- Monotonicity: If $\mathbf{p} \geq \mathbf{p}^{\prime}$, then $\mathbf{I}(\mathbf{p}) \geq \mathbf{I}\left(\mathbf{p}^{\prime}\right)$.

- Scalability: For all $\alpha>1, \alpha \mathbf{I}(\mathbf{p})>\mathbf{I}(\alpha \mathbf{p})$.

The main convergence result for standard interference functions can be summarized as follows:

Proposition 2 ( [5]) Let $\mathbf{I}(\mathbf{p})$ be a standard interference function and consider the iteration

$$
\mathbf{p}(n+1)=\mathbf{I}(\mathbf{p}(n))
$$

Then, if (3) has a fixed-point, this fixed-point is unique and the iterates $\{\mathbf{p}(n)\}$ produced by (3) converge to the fixed-point from any initial vector $\mathbf{p}(0)$.

Note that contrary to the result for contraction mappings, the existence of fixed-points has to be verified separately, and no guarantees about the convergence rate of the iterates to the fixed-point are given. Already this should raise the suspicion that standard interference function do not define contraction mappings in normed linear spaces. The following simple example establishes that this suspicion is indeed correct.

\section{Example 1 (Interference functions are not contractive)}

Consider the function

$$
\mathbf{I}(\mathbf{p})=2 \mathbf{p}+\mathbf{1}
$$

where 1 is the vector with all components equal to 1 . This is a standard interference function, but $\left\|\mathbf{I}(\mathbf{p})-\mathbf{I}\left(\mathbf{p}^{\prime}\right)\right\|=2 \| \mathbf{p}-$ $\mathbf{p}^{\prime} \|$, so it is neither contractive nor para-contractive.

To establish convergence rates of power control algorithms, one could certainly make a separate analysis of contractivity of the particular interference functions at hand. However, if one can prove contractivity, particularly in the maximum norm, then the interference function framework brings little additional value. The beauty of the framework lies in the easily verifiable conditions that guarantee synchronous and asynchronous convergence. Next, we will show that a new definition of the scalability condition ensures contractivity.

\section{CONTRACTIVE INTERFERENCE FUNCTIONS}

We propose to study a different class of functions which we call contractive interference functions.

Definition 2 A function $\mathbf{I}(\mathbf{p})$ is said to be a contractive interference function if it, for all $\mathbf{p} \geq \mathbf{0}$, satisfies the following conditions:

- Positivity: $\mathbf{I}(\mathbf{p})>\mathbf{0}$.

- Monotonicity: If $\mathbf{p} \geq \mathbf{p}^{\prime}$, then $\mathbf{I}(\mathbf{p}) \geq \mathbf{I}\left(\mathbf{p}^{\prime}\right)$.

- Contractivity: There exists a positive vector $\mathbf{v}$ and $a$ constant $c \in[0,1)$ such that for all $\epsilon>0, \mathbf{I}(\mathbf{p}+\epsilon \mathbf{v}) \leq$ $\mathbf{I}(\mathbf{p})+c \epsilon \mathbf{v}$.

Note that the two first conditions are the same as for standard interferernce functions, but the scalability condition has now been replaced by contractivity. As shown in the next theorem, contractive interference functions define contraction mappings, which implies that the associated iterations (3) have unique fixed-points and geometric convergence rates.

Theorem 1 If $\mathbf{I}(\mathbf{p})$ is a contractive interference function, then (3) has a unique fixed point $\mathbf{p}^{\star}$ and the iterates $\{\mathbf{p}(n)\}$ produced by (3) converge geometrically to $\mathbf{p}^{\star}$ from every initial vector $\mathbf{p}(0)$.

Proof: Let $\mathbf{p} \neq \mathbf{p}^{\prime}$, then $\mathbf{p} \leq \mathbf{p}^{\prime}+\left\|\mathbf{p}-\mathbf{p}^{\prime}\right\|_{\infty}^{v} \mathbf{v}$. Since $\left\|\mathbf{p}-\mathbf{p}^{\prime}\right\|_{\infty}^{v}>0$, the monotonicity and contractivity properties imply

$$
\mathbf{I}(\mathbf{p}) \leq \mathbf{I}\left(\mathbf{p}^{\prime}\right)+c\left\|\mathbf{p}-\mathbf{p}^{\prime}\right\|_{\infty}^{v} \mathbf{v}
$$

By interchanging role of $\mathbf{p}$ and $\mathbf{p}^{\prime}$,

$$
\mathbf{I}\left(\mathbf{p}^{\prime}\right) \leq \mathbf{I}(\mathbf{p})+c\left\|\mathbf{p}-\mathbf{p}^{\prime}\right\|_{\infty}^{v} \mathbf{v}
$$

So for all components of $\mathbf{I}(\mathbf{p})$, we have

$$
\left|I_{i}(\mathbf{p})-I_{i}\left(\mathbf{p}^{\prime}\right)\right| \leq c\left\|\mathbf{p}-\mathbf{p}^{\prime}\right\|_{\infty}^{v} v_{i} \forall i
$$


Therefore, $\left\|\mathbf{I}\left(\mathbf{p}^{\prime}\right)-\mathbf{I}(\mathbf{p})\right\|_{\infty}^{v} \leq c\left\|\mathbf{p}-\mathbf{p}^{\prime}\right\|_{\infty}^{v}$. From Proposition $1, \mathbf{I}(\mathbf{p})$ is contractive and hence it has a unique fixed point. Moreover for every initial vector $\mathbf{p}$, the sequence $\mathbf{p}(n+1)=$ $\mathbf{I}(\mathbf{p}(n))$ converges to $\mathbf{p}^{\star}$ geometrically.

To emphasize the modulus $c$ of the contraction mapping, we sometimes say that a function is a c-contractive interference function. To show that the concept of contractive interference function is useful, we will now show that it is readily applied to several distributed power control algorithms from the literature yielding, sometimes for the first time, guaranteed convergence rates for these algorithms. We denote by $\mathcal{T}$ the set of transmitters and $\mathcal{R}$ the set of receivers in the network.

Firstly, we consider linear interference functions:

$$
I_{i}(\mathbf{p})=\gamma_{i} \frac{\sum_{j \in \mathcal{T}, j \neq i} G_{i j} p_{j}+\eta_{i}}{G_{i i}}, \quad i \in \mathcal{R},
$$

where $G=\left[G_{i j}\right] \geq 0$ is a fixed link gain matrix, $\gamma_{i}$ and $\eta_{i}$ are the target SINR and the background noise at the receiver of transmitter $i$, respectively. This equation can be rewritten as $I_{i}(\mathbf{p})=\sum_{j \in \mathcal{T}, j \neq i}^{K} M_{i j} p_{j}+N_{i}, i \in \mathcal{R}$, where $N_{i}=\gamma_{i} \eta_{i} / G_{i i}$ and

$$
M_{i j}= \begin{cases}\gamma_{i} G_{i j} / G_{i i} & , j \neq i \\ 0 & , j=i .\end{cases}
$$

We then have the following result.

Theorem 2 If $\|M\|_{\infty}^{v}<1$ for some $\mathbf{v}>0$, the linear interference functions (4) are c-contractive interference functions with $c=\|M\|_{\infty}^{v}$.

Proof: It is clear that linear interference functions are positive and monotone. Furthermore,

$$
\mathbf{I}(\mathbf{p}+\epsilon \mathbf{v})=M(\mathbf{p}+\epsilon \mathbf{v})+N \leq \mathbf{I}(\mathbf{p})+\|M\|_{\infty}^{v} \epsilon \mathbf{v} .
$$

Hence, $\mathbf{I}(\mathbf{p})$ is contractive with $c=\|M\|_{\infty}^{v}$.

Since $M$ is a square nonnegative matrix, $\rho(M)<1$ is equivalent to the existence of a positive vector $\mathbf{v}$ for which $\|M\|_{\infty}^{v}<1$ [14, pp.148]. In this case, $\mathbf{v}$ is the right PerronFrobenius eigenvector of $M$. The same analysis with $\mathbf{v}=\mathbf{1}$ (which corresponds to an unweighted max-norm contraction) for the linear interference functions would yield $\|M\|_{\infty}<1$ which is a sufficient condition for convergence of (3), but conservative since $\rho(M) \leq\|M\|_{\infty}$.

One can carry out a similar analysis also for the minimum power assignment and macro-diversity interference functions from [5], but the poofs are omitted here for brevity.

To show that our framework allows to go beyond the known results, consider the utility-based power control (UBPC) from [9]. The associated interference function is

$$
I_{i}^{u}(\mathbf{p})=\left(\frac{\sum_{i \neq j} G_{i j} p_{j}+\eta_{i}}{G_{i i}}\right) f_{i}^{-1}\left(\alpha_{i} \frac{\sum_{i \neq j} G_{i j} p_{j}+\eta_{i}}{G_{i i}}\right)
$$

where $\alpha_{i}$ is a price coefficient and $f_{i}^{-1}(x)$ is a decreasing function on $\left[\underline{K}_{i}, \bar{K}_{i}\right]$ for all $i$ given by

$$
f_{i}\left(S I R_{i}\right)=U_{i}^{\prime}\left(S I R_{i}\right)
$$

where $U_{i}$ is a utility function of user $i$. In their paper, Xiao et al. use a sigmoidal utility function

$$
U_{i}\left(S I R_{i}\right)=\frac{1}{1+e^{-a_{i}\left(S I R_{i}-b_{i}\right)}}
$$

where $b_{i}=\gamma_{i}-a_{i}^{-1} \ln \left(a_{i} \gamma_{i}-1\right)$. Let $M_{i j}$ be defined as (5). We will next show that the framework of contractive interference functions will allow us to analytically bound the convergence rate, which has an immediate use for tuning the algorithm parameters. Specifically, we have the following result.

Theorem 3 Consider the interference function $I^{u}(p)=$ $\left[I_{i}^{u}(p)\right]$ defined in (6-8). If $c=\max _{i}\left\{b_{i} \sum_{j=1}^{K} M_{i j}\right\}<1$ then $I^{u}(p)$ is a c-contractive interference function.

Proof: Let $\mathbf{v}=\mathbf{1}$. For all $\epsilon>0$ we have

$$
\begin{aligned}
I_{i}^{u}(\mathbf{p}+\epsilon \mathbf{1}) & =\left(\sum_{j=1}^{K} M_{i j}\left(p_{j}+\epsilon\right)+N_{i}\right) \\
f_{i}^{-1}\left(\alpha_{i}\left(\sum_{j=1}^{K} M_{i j}\left(p_{j}+\epsilon\right)+N_{i}\right)\right) & \left(\sum_{j=1}^{K} M_{i j}\left(p_{j}+\epsilon\right)+N_{i}\right) \\
& f_{i}^{-1}\left(\alpha_{i}\left(\sum_{j=1}^{K} M_{i j} p_{j}+N_{i}\right)\right) \\
& =I_{i}^{u}(\mathbf{p})+\epsilon\left(\sum_{j=1}^{K} M_{i j}\right) f_{i}^{-1}\left(\alpha_{i}\left(\sum_{j=1}^{K} M_{i j} p_{j}+N_{i}\right)\right)
\end{aligned}
$$

where the first inequality comes from the fact that $f_{i}^{-1}(x)$ is a decreasing function. Since the maximum value of $f_{i}^{-1}(x)$ on $\left[\underline{K}_{i}, \bar{K}_{i}\right]$ is $f_{i}^{-1}\left(\underline{K}_{i}\right)$, if $\max _{i}\left\{f_{i}^{-1}\left(\underline{K}_{i}\right)\left(\sum_{j=1}^{K} M_{i j}\right)\right\}<1$, then UBPC iteration geometrically converges to a unique fixed point. Under the sigmoidal utility function (8), the maximum value of $U_{i}^{\prime}\left(S I R_{i}\right)$ occurs at point $b_{i}$, and we have $\max f_{i}^{-1}(x)=b_{i}$. Hence, $f_{i}^{-1}\left(\underline{K}_{i}\right) \leq b_{i}$ and the result follows.

The following numerical example illustrates our result.

Example 2 We consider UBPC under the simulation scenario described in [9]. Here, four mobiles share a channel with link gain matrix $G$ given by

$$
G=\left[\begin{array}{cccc}
10^{-4} & 6.82 \times 10^{-7} & 3.57 \times 10^{-8} & 2.12 \times 10^{-8} \\
1.52 \times 10^{-7} & 6.25 \times 10^{-4} & 3.51 \times 10^{-6} & 1.98 \times 10^{-7} \\
7.67 \times 10^{-9} & 2.44 \times 10^{-8} & 1.23 \times 10^{-6} & 5.16 \times 10^{-9} \\
2.63 \times 10^{-7} & 4.82 \times 10^{-8} & 2.56 \times 10^{-7} & 3.28 \times 10^{-5}
\end{array}\right]
$$

The noise power is 0.5 and the target SIRs of the users are $6,6,8$ and $10 \mathrm{~dB}$, respectively. We assume that four users in the system use sigmoidal utility function with parameters $1.02,1.32,0.88$ and 1.05, respectively. The price coefficient $\alpha$ is equal to 5000 for all users. Fig. 1 shows the norm between power vector and optimal power vector with respect to iteration. The upper bound obtained from proposition 8 is plotted as a dotted curve. 


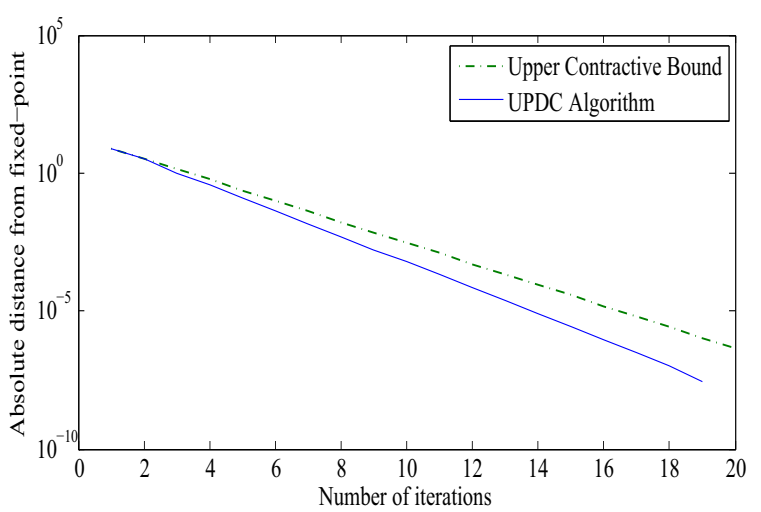

Fig. 1. Comparison of upper bound on convergence rate of UBPC obtained in Proposition 8 and the actual convergence rate of UBPC for the scenario considered in example 2.

Another useful result shows that imposing an upper and lower bound on a contractive interference function does not change the contractivity properties.

Theorem 4 If $\mathbf{I}(\mathbf{p})$ is a contractive interference function, then so is $\mathbf{I}^{q}(\mathbf{p})=\max \left\{\mathbf{p}_{\min }, \min \left\{\mathbf{p}_{\max }, \mathbf{I}(\mathbf{p})\right\}\right\}$.

Proof: Omitted for brevity but can be found in [13].

\section{INTERFERENCE FUNCTIONS AND PARA-CONTRACTIONS}

We have already shown that standard interference functions do not define contraction mappings. However, the convergence results for standard interference functions are identical to those of para-contractions, so there should be a link between the two. This section shows one such link. In particular, we demonstrate that a logarithmic change of variables $\mathbf{s}=\ln (\mathbf{p})$ makes the iterations para-contracting in the new variables.

Theorem 5 Suppose that the interference function $\mathbf{I}(\mathbf{p})$ is standard. Then the change of variables $\mathbf{s}=\ln (\mathbf{p})$ and $\widetilde{\mathbf{I}}(\mathbf{s})=\ln \left(\mathbf{I}\left(\mathbf{e}^{\mathbf{s}}\right)\right)$ transforms the interference function $\mathbf{I}(\mathbf{p})$ into a para-contracting function $\widetilde{\mathbf{I}}(\mathbf{s})$.

Proof: First we rewrite the properties of standard interference function in the new coordinates.

1) monotonicity: If $\mathbf{s}_{1} \leq \mathbf{s}_{2}$, then $\widetilde{\mathbf{I}}\left(\mathbf{s}_{1}\right) \leq \widetilde{\mathbf{I}}\left(\mathbf{s}_{2}\right)$.

2) sub-additivity: For all $\epsilon>0, \widetilde{\mathbf{I}}(\mathbf{s}+\epsilon \overline{\mathbf{1}})<\epsilon \mathbf{1}+\widetilde{\mathbf{I}}(\mathbf{s})$.

Let $\mathbf{s}_{1} \neq \mathbf{s}_{2}$, then monotonicity and sub-additivity of $\widetilde{\mathbf{I}}(\mathbf{s})$ imply

$$
\left\|\widetilde{\mathbf{I}}\left(\mathbf{s}_{1}\right)-\widetilde{\mathbf{I}}\left(\mathbf{s}_{2}\right)\right\|_{\infty}<\left\|\mathbf{s}_{1}-\mathbf{s}_{2}\right\|_{\infty}
$$

Therefore, $\widetilde{\mathbf{I}}(\mathbf{s})$ is a para-contraction. Due to space constraints, we refer reader to [13] for a detailed proof.

Theorem 5 helps us to understand that interference functions are para-contractions with respect to a certain metric space. Specifically, we note the following:
Theorem 6 Standard interference functions are para-contractions with respect to the metric space $d_{c}\left(\mathbf{p}, \mathbf{p}^{\prime}\right)=\max _{i}\left|\ln \frac{p_{i}}{p_{i}^{\prime}}\right|$.

It is important to note that standard interference functions are para-contractions on the metric space induced by $d_{c}$ irrespectively if they have a fixed-point or not. To guarantee convergence of the iterates, we must verify that the iteration has fixed-points. The following theorem can then be useful.

Theorem 7 Given a standard interference function $\mathbf{I}(\mathbf{p})$, if there exists a $\mathbf{p}^{\prime}$ such that $\mathbf{I}\left(\mathbf{p}^{\prime}\right) \leq \mathbf{p}^{\prime}$, then a fixed point exists.

\section{TWO-SIDED INTERFERENCE FUNCTIONS}

Sung and Leung [10] present a new class of functions called two-sided scalable interference functions which generalizes the standard interference functions to allow for simple and powerful analysis of certain opportunistic power control laws:

Definition 3 (Two-sided scalable interference functions [10]) A function $\mathbf{I}(\mathbf{p})$ is called two-sided scalable interference function, if for all $\mathbf{p} \geq \mathbf{0}, \mathbf{I}(\mathbf{p})$ satisfies:

- Positivity: $\mathbf{I}(\mathbf{p})>\mathbf{0}$, and

- Two-sided scalability: For all $\alpha>1$,

$$
\frac{1}{\alpha} \mathbf{p} \leq \mathbf{p}^{\prime} \leq \alpha \mathbf{p} \Rightarrow \frac{1}{\alpha} \mathbf{I}(\mathbf{p})<\mathbf{p}^{\prime}<\alpha \mathbf{I}(\mathbf{p}) .
$$

Note how monotonicity and scalability has been replaced by the two-sided scalability condition. The key convergence result reads as follows:

Proposition 3 ( [10]) Let $\mathbf{I}(\mathbf{p})$ be a two-sided scalable interference function and consider the iteration (3). If the iteration has a fixed-point $p^{\star}$, then this fixed-point is unique and the sequence $\{\mathbf{p}(n)\}$ generated by the iteration converges to $p^{\star}$ for every initial value $\mathbf{p}(0)$.

The convergence conditions for two-sided scalable interference functions coincide with those of para-contractions. Our framework introduces the two-sided contractive interference functions with which we can guarantee existence and uniqueness of fixed points along with convergence rates.

Definition 4 A function $\mathbf{I}(\mathbf{p})$ is called two-sided contractive interference function if it, for all $\mathbf{p} \geq \mathbf{0}$ satisfies

- Positivity: $\mathbf{I}(\mathbf{p})>\mathbf{0}$, and

- Two-sided contractivity: there exists a positive vector $\mathbf{v}$ and a constant $c \in[0,1)$ such that for all $\epsilon>0, \mathbf{p}^{\prime}-\epsilon \mathbf{V} \leq$ $\mathbf{p} \leq \mathbf{p}^{\prime}+\epsilon \mathbf{v}$ implies that

$$
I\left(\mathbf{p}^{\prime}\right)-c \epsilon \mathbf{v} \leq \mathbf{I}(\mathbf{p}) \leq \mathbf{I}\left(\mathbf{p}^{\prime}\right)+c \epsilon \mathbf{v} \text {. }
$$

Theorem 8 If $\mathbf{I}(\mathbf{p})$ is a two-sided contractive interference function, then (3) has a unique fixed point $\mathbf{p}^{\star}$ and the sequence $\{\mathbf{p}(n)\}$ generated by the iteration (3) converges geometrically to $\mathbf{p}^{\star}$ from every initial value $\mathbf{p}(0)$. 
The proof follows similarly to the contractive interference function proof and is omitted in this paper. Next, we show how two-sided scalable functions relate to para-contractions.

Theorem 9 Suppose that $\mathbf{I}(\mathbf{p})$ is two-sided scalable. Then $\mathbf{I}(\mathbf{p})$ is a para-contraction in a metric space defined by $d_{c}\left(\mathbf{p}, \mathbf{p}^{\prime}\right)=\max _{i}\left|\ln \frac{p_{i}}{p_{i}^{\prime}}\right|$.

Our results in this section are related to the work by Möller and Jönsson [12], [15], who studied stability of higher-order power control laws. They demonstrated that in logarithmic variables, two-sided scalability implies global Lipschitz continuity of the interference function, and an alternative restriction allows to establish convergence rates and uniqueness of fixedpoints. The link to paracontractions, although not phrased this way, can also be seen in [12, Proposition 3].

\section{Vi. Totaly Asynchronous Power Control}

So far, we have examined synchronous power control algorithms. In this case, every component of the vector $\mathbf{p}$ is updated at every time step, using information of the transmit powers used by all transmitters in the previous iteration. However, a nice feature of the stadard interference functions is that they also converge when executed asynchronously. In this section, we will demonstrate that contractive interference functions also converge asynchronously.

To state the precise result, we recall the totally asynchronous computational model from Bertsekas and Tsitsiklis [14, pp. 426]. Let $T$ be the set of times when some transmitter updates its power, and let $T^{i} \subseteq T$ be the times when transmitter $i$ executes an update. To model that the transmitter might need to update its power using old information from other transmitters, let $\tau_{j}^{i}(t)$ be the time at which the most recent version of $p_{j}$ available to node $i$ at time $t$ was computed. Node $i$ executes the update

$$
p_{i}(t+1)= \begin{cases}I_{i}\left(p_{1}\left(\tau_{1}^{i}(t)\right), \cdots, p_{n}\left(\tau_{n}^{i}(t)\right)\right) & \forall t \in T^{i} \\ p_{i}(t) & \forall t \notin T^{i}\end{cases}
$$

Definition 5 (Total Asynchronism [14, pp. 431]) The iteration (10) is totally asynchronous if the sets $T^{i}$ are infinite for all $i$, and if $\left\{t_{k}\right\}$ is a sequence of elements of $T^{i}$ that tends to infinity, then it also holds that $\lim _{k \rightarrow \infty} \tau_{j}^{i}\left(t_{k}\right)=\infty \forall j$

Loosely speaking, this assumption guarantees that no transmitter ceases to update its power, and that such updates eventually propagate to all other transmitters in the network. Yates showed that if an iteration involving standard interference function converges synchronously, it also converges when executed totally asynchronously. A similar result holds for contracting interference functions:

Theorem 10 Let $\mathbf{I}(\mathbf{p})$ be a contractive interference function. Then, the iterates produced by (10) converge to the unique fixed-point under total asynchronism.

This result is proven by noticing that contractive interference functions define max-norm contractions, and that maxnorm contractions converge under total asynchronism (e.g. [14, pp. 434]). A similar result can be established for two-sided contractive interference functions using the same arguments.

\section{CONCLUSIONS}

In this paper we have shown that standard interference functions do not define contraction mappings and introduced contractive interference functions that guarantee existence and uniqueness of fixed-point along with geometric convergence of iterates. We have demonstrated that several important distributed power control algorithms proposed in the literature are contractive and derived the associated convergence rates. In some cases, such as linear iterations, the convergence rate coincides with known results from the literature that has been obtained using a detailed and tailored analysis. In other cases, such as the utility-based power control, we provide the first convergence rate estimates in the literature. This paper also clarifies the link between standard interference functions and para-contractions.We have also shown that contractive interference functions converge when executed totally asynchronously.

\section{REFERENCES}

[1] G. J. Foschini and Z. Miljanic, "A simple distributed autonomous power control algorithm and its convergence," IEEE Transactions on Vehicular Technology, vol. 42, pp. 641-646, 1993.

[2] J. Zander, "Distributed cochannel interference control in cellular radio systems," IEEE Transactions on Vehicular Technology, vol. 41, no. 3, pp. 305-311, 1992.

[3] D. Mitra, "An asynchronous distributed algorithm for power control in cellular radio systems," Proceedings of the 1993 WINLAB Workshop, pp. 249-259, 1993

[4] F. Berggren, "Power control and adaptive resource allocation in DSCDMA," Ph.D. dissertation, KTH, Stockholm, Sweden, 2003.

[5] R. Yates, "A framework for uplink power control in cellular radio systems," IEEE Journal on Selected Areas in Communications, vol. 13, no. 7, pp. 1341-1347, 1995.

[6] C.-Y. Huang and R. Yates, "Rate of convergence for minimum power assignment algorithms in cellular radio systems," Wireless Networks, vol. 4, no. 4, pp. 223-231, 1998 .

[7] J. Herdtner and E. Chong, "Analysis of a class of distributed asynchronous power control algorithms for cellular wireless systems," IEEE Journal on Selected Areas in Communications, vol. 18, no. 3, pp. 436446, 2000.

[8] M. Chen and D. Tse, "An upper bound on the convergence rate of uplink power control in DS-CDMA systems," IEEE Communications Letters, vol. 10, no. 4, pp. 231-233, 2006

[9] M. Xiao, N. Shroff, and E. Chong, "A utility-based power-control scheme in wireless cellular systems," IEEE Journal on Selected Areas in Communications, vol. 11, no. 2, pp. 210-221, 2003.

[10] C. Sung and K. Leung, "A generalized framework for distributed power control in wireless networks," IEEE Transactions on Information Theory, vol. 51, no. 7, pp. 2625-2635, 2005.

[11] M. Schubert and H. Boche, "A generic approach to QoS-based transceiver optimization," IEEE Transactions on Communications, vol. 55, no. 8, pp. 1557-1566, 2007.

[12] A. Möller and U. Jönsson, "Stability of high order distributed power control," in $48^{\text {th }}$ IEEE Conference on Decision and Control, 2009, pp. 4963-4970.

[13] T. C. H. R. Feyzmahdavian and M. Johansson, "Contractive interference functions and rates of convergence of distributed power control laws," Available: http://arxiv.org/abs/1201.3740, 2012.

[14] D. P. Bertsekas and J. N. Tsitsiklis, Parallel and Distributed Computation. Prentice-Hall, 1989.

[15] A. Möller and U. Jönsson, "Input output analysis of power control in wireless networks," in $49^{\text {th }}$ IEEE Conference on Decision and Control, 2010, pp. 6451-6456. 